Horizons philosophiques

\title{
Les transcendantalistes : indépendance et infini
}

\section{Sylvie Chaput}

Volume 2, numéro 1, automne 1991

De Buenos Aires à Québec

URI : https://id.erudit.org/iderudit/800888ar

DOI : https://doi.org/10.7202/800888ar

Aller au sommaire du numéro

Éditeur(s)

Collège Édouard-Montpetit

ISSN

1181-9227 (imprimé)

1920-2954 (numérique)

Découvrir la revue

Citer cet article

Chaput, S. (1991). Les transcendantalistes : indépendance et infini. Horizons philosophiques, 2(1), 107-114. https://doi.org/10.7202/800888ar d'utilisation que vous pouvez consulter en ligne.

https://apropos.erudit.org/fr/usagers/politique-dutilisation/ 


\section{Les transcendantalistes: indépendance et infini}

Ermite, prisonnier d'opinion : c'est à ce double titre que Henry David Thoreau est célèbre. Que sa captivité n'ait duré qu'une nuit, qu'il ait séjourné seulement deux ans dans une cabane, que l'étang au bord duquel elle se trouvait ait été non pas loin de toute civilisation, mais près de Concord où Thoreau comptait parents et amis, on est toujours un peu déçu ou fâché de l'apprendre. Mais, parmi les philosophes des trois Amériques, ceux qui sont entrés dans la légende sont si rares, que nous serions malvenus de rester sur un mouvement d'humeur. Tout bien considéré, d'ailleurs, la réputation de cette cabane et de cette prison n'est pas surfaite.

Thoreau a eu l'amabilité de résumer sa philosophie. Elle tient en trois mots, ou plutôt en un seul mot répété trois fois : "Simplifier, simplifier, simplifier». Une autre phrase de Walden exprime un message identique avec humour : "Le fermier essaye de résoudre le problème de la vie à gagner par une formule plus compliquée que le problème lui-même; pour se procurer des lacets de souliers, il spécule en termes de troupeaux de bêtes ${ }^{1} . " \mathrm{Ce}$ système, auquel nous n'avons toujours pas échappé et que

1. Henry David Thoreau, Walden ou la vie dans les bois, trad. G: LandréLaugier, Paris, Aubier, collection bilingue, 1967, p. 115. 
nous continuons de nourrir, Thoreau en a montré et dénoncé l'absurdité avec une rare persévérance. Ses moyens étaient modestes; il a tenu à ce qu'ils le demeurent, exerçant tour à tour les métiers d'instituteur, de fabricant de crayons, d'homme à tout faire et d'arpenteur. Dire qu'il s'efforçait de réduire ses besoins au minimum serait erroné. Au contraire, c'était en fonction de ses besoins primordiaux - avoir du temps, marcher, observer, méditer, être luimême - qu'il organisait sa vie.

Thoreau réprouvait la division du travail. «Sans doute, écrivait-il, un autre peut penser à ma place; mais ce n'est pas une raison pour qu'il soit souhaitable qu'il le fasse, pour que moi-même je cesse de le faire ${ }^{2}$." Peu soucieux du qu'en-dira-t-on, réfractaire à toute recherche de l'effet, il détestait les ornements architecturaux : «Ce que je peux découvrir à présent, en fait de beauté, en architecture, s'est graduellement développé du dedans au dehors, venant des besoins et de la nature de l'occupant $[. . .]^{3}$."

"Graduellement développé du dedans au dehors": ces mots sont lourds de sens. Quand Thoreau construit sa cabane, en 1845, il répond à une nécessité intérieure et crée le territoire qui sera le sien. II commence à vivre comme il l'entend, à l'écart du bruit et des nouvelles "Read not the Times, disait-il. Read the Eternities 4 ». II se place en position de limiter les intrusions, de filtrer les visiteurs, de les retenir ou non à son gré. La première fonction de la maison, selon lui, est de préserver la chaleur, et il n'est pas homme à mal joindre les planches. Mais dans cette retraite, c'est aussi sa propre chaleur qu'il préserve, lui-même qu'il bâtit.

2. Ibid., p. 133.

3. Ibid.

4. "Life Without Principle", Walden and Other Writings of Henry David Thoreau, Brooks Atkinson ed., The Modern Library, 1965 (1937), p. 727. 
Lorsque, dans la conférence qui deviendra La désobéissance civile ${ }^{5}$, il expliquera à ses concitoyens pourquoi il a refusé de payer son impôt en 1846 et racontera son emprisonnement, il se moquera d'un appareil judiciaire qui prétendait l'enfermer alors que son esprit pouvait traverser les murs et voyager librement.

La cabane prépare la prison. Elle est une première résistance à l'entourage. Elle est le lieu d'une expérience de construction de soi, de confiance en soi, d'affirmation de soi. Sûr de ce qu'il pense, Thoreau pourra s'opposer, le moment venu, à une loi injuste, s'indigner de ce que l'État prenne pour acquis qu'il accepte d'encourager l'esclavage, dire : "Non, voilà les bornes que vous ne pouvez pas dépasser." Ancré en lui-même, il pourra, en esprit, traverser les murs de sa cellule, tout comme, de sa cabane, il part explorer la forêt. Thoreau est celui qui, infatigablement, passe au crible les pouvoirs qui cherchent à s'imposer à lui, se concentre pour trouver sa propre pensée, et se remet en route.

On convient que le mouvement transcendantaliste est né à la suite d'une rencontre de ministres et d'ex-ministres unitariens qui s'est tenue à Boston en 1836. Emerson faisait partie de ces ex-pasteurs. Je m'arrêterai maintenant au "Discours de l'École de théologie", qu'il a prononcé en 1838 et à cause duquel cette école, celle de Harvard, ne l'a plus invité durant trente ans.

Contrairement aux calvinistes, les unitariens ne croyaient pas en la Trinité, en la divinité du Christ ni en la prédestination. Emerson n'y croyait pas non plus, mais il avait abandonné l'unitarisme, pour diverses raisons. II re-

5. Henry David Thoreau, La désobéissance civile, suivi de "Visiteurs", chapitre 6 de Walden, traduction, introduction et notes de $S$. Chaput, postface de Marc Chabot, Montréal, L'Hexagone/Minerve, coll. Balises, 1982, 138 p. 
prochait, par exemple, à ses anciens collègues de négliger leurs fidèles pour livrer des combats d'exégèse aux ministres calvinistes (surtout au sujet des miracles relatés dans la Bible) et, globalement, de sacrifier l'esprit à la lettre.

Les arguments du «Discours de l'École de théologie» sont les suivants : $1^{\circ}$ La vérité ne s'enseigne pas; elle est saisie par une intuition, le sentiment moral. $2^{\circ}$ L'homme est divin («Si un homme porte en son cœur la justice, alors il est Dieu», disait Emerson; l'Être suprême habite en l'homme.) $3^{\circ}$ C'est une erreur de vouer un culte à la personne du Christ, de le considérer comme Dieu : "Jésus appartenait à la vraie race des prophètes. II voyait distinctement le mystère de l'âme. [...] Seul dans toute l'histoire, il a apprécié à sa juste mesure la grandeur de l'homme. Un homme a été fidèle à ce qui est en vous et en moi. II a vu que Dieu s'incarne en l'homme». $4^{\circ}$ Les Églises chrétiennes ont tort de présenter la révélation comme quelque chose de terminé - comme si Dieu était mort. Les fidèles et les ministres (Emerson s'adressait à des aspirants au pastorat) doivent chercher, au-delà des rites et des sermons, à nouer un rapport direct avec Dieu : «En l'âme, dit-il, cherchons la rédemption. Partout où survient un homme, la révolution l'accompagne. L'ancien est bon pour les esclaves. [...] Le faiseur de merveilles, c'est l'homme. "l est entouré de miracles ${ }^{6} . "$

En 1845, Margaret Fuller publiait La femme au dixneuvième siècle. De cet ouvrage qui est à la fois l'un des premiers essais féministes américains et le seul de tous les livres transcendantalistes qui porte spécifiquement sur

6. Le texte intégral du “Discours de l'École de théologien («The Divinity School Address») se trouve dans plusieurs recueils anglais d'Emerson, par exemple aux pages 100-116 de : Selections from Ralph Waldo Emerson, Boston, S.E. Whicher ed., Riverside Edition, 1957. 
les rapports femmes-hommes, je ne retiendrai, ici, qu'un passage sur le célibat. Après avoir affirmé que les femmes devraient cesser de prendre des hommes comme maîtres et comme guides, parce que, malgré leur bonne volonté, ils sont trop attachés aux traditions pour les aider à trouver leur voie, elle écrit ceci :

Si quelque individu vit trop de ses relations, de sorte que les ressources de sa propre nature lui deviennent étrangères, il tombe, au bout d'un moment, dans un état de démence ou d'imbécillité dont seul un temps d'isolement peut le guérir, un temps qui permet aux fontaines régénératrices de jaillir. Pour une société, il en va de même. Bien des esprits, privés des moyens traditionnels ou instinctifs de mener une existence heureuse, doivent trouver du secours dans leurs propres impulsions, sans quoi ils périssent. C'est pourquoi, bien qu'il faille accueillir avec joie toute élévation en vue de l'union, nous ne refusons pas au célibat le titre de fait le plus marquant de notre époque. [...] Pour l'instant, les rameurs déposent leurs rames; ils attendent un changement avant de pouvoir ramer ensemble. Tout concourt à illustrer la pensée d'un sage contemporain : l'union n'est possible que pour ceux qui sont des unités ${ }^{7}$.

Je soulèverai un dernier point avant de conclure. Alors que Thoreau quitta les États-Unis seulement une fois pour passer huit jours au Québec, Margaret Fuller fut européenne d'adoption pendant les quatre dernières années de sa vie. Partie de New York comme journaliste en 1846, elle se trouva plongée dans les révolutions de 1848 et adhéra à la cause italienne. Être dans l'histoire, voir l'histoire se faire, la passionnait davantage que ses deux compagnons. Pourtant, elle demeurait très Américaine. Elle souhaitait la chute des monarchies européennes parce qu'elle était convaincue que la république était la meilleure forme de gouvernement. C'était en rappelant à ses com-

7. Margaret Fuller, La femme au dix-neuvième siècle, trad. S. Chaput, Montréal, Éditions Saint-Martin, 1988, p. 95-96. 
patriotes leurs idéaux d'égalité, de liberté et de justice qu'elle tentait, dans ses articles, de les convaincre de soutenir concrètement l'ttalie. Mais la plupart d'entre eux restaient sourds à ses appels. Les Américains acceptaient l'esclavage, s'orientaient vers une politique expansionniste, s'enlisaient dans la recherche du profit. C'est pourquoi elle refusait de rentrer.

En Europe, au milieu des enseignements de l'adversité, écrivait-elle, lutte un esprit plus noble, - un esprit qui réjouit et anime le mien. J'entends des paroles sincères, qui témoignent d'une foi et d'un amour purs. Je vois des gestes de fraternité. Voilà ce qui fait mon Amérique ${ }^{8}$.

Thoreau, avait peut-être quitté sa cabane au bout de deux ans parce qu'elle s'était confondue avec lui, parce qu'il n'avait plus besoin de cette coquille. Margaret Fuller, elle, retrouvait outre-mer le pays qu'elle portait en ellemême. L'esprit plutôt que la lettre, comme insistait Emerson.

Le courant transcendantaliste est né soixante ans après la Déclaration d'indépendance des États-Unis. Son apparition signale une volonté de poursuivre, sur le terrain philosophique et littéraire, ce qui était déjà accompli sur le terrain politique. En 1837, dans L'intellectuel américain, Emerson insistait sur la nécessité, pour les Américains, de cesser d'écouter les muses de l'Europe - sur la nécessité de marcher sur leurs propres jambes, de travailler de leurs propres mains, d'exprimer leurs propres pensées. À cette

8. Margaret Fuller Ossoli, At Home and Abroad, or Things and Thoughts in America and Europe, New York, Arthur B. Fuller ed., Kennikat Press, Port Washington, 1971 (réimpr. de l'édition originale de 1856), p. 327, cité en français dans S. Chaput, Margaret Fuller (1810-1850), Montréal, Éditions Saint-Martin, 1988, p. 197. 
idée d'une philosophie et, surtout, d'une littérature authentiquement américaines - idée qui n'était pas nouvelle et qu'ils n'étaient pas les seuls à défendre, les transcendantalistes ajoutaient autre chose. La conviction que tout être humain possède des capacités illimitées, qu'il est profondément original, qu'il a son génie propre, qu'il doit avoir la possibilité de développer pleinement sa propre nature et que c'est là son devoir, sa mission.

On pourrait dire que le transcendantalisme est authentiquement américain par l'accent qu'il met sur la confiance en soi et sur le développement de soi. On pourrait même dire, avec un brin d'ironie, qu'il fallait bien des Américains pour avoir la prétention de dire que les êtres humains sont divins - ou la naïveté d'affirmer, comme Emerson, Thoreau et Fuller l'ont souvent fait, que le mal n'est qu'une apparence, une sorte de fantôme devant lequel il suffit de se tenir debout pour le faire disparaître. On pourrait noter au passage que Thoreau, en refusant de payer un impôt pour marquer son indépendance par rapport à l'État, rejouait, seul, un épisode collectif de l'histoire des États-Unis, le Boston Tea Party. Tout cela serait juste.

Pourtant, en cherchant le point de vue à partir duquel je vous parlerais ce matin, quelque chose m'est apparu, que je n'avais pas constaté auparavant. Sans doute, j'ai aperçu cela parce que, pour la première fois, je plaçais Thoreau, Emerson et Fuller devant moi sur le même plan, et parce que le thème de notre table ronde est indéniablement relié à la géographie, c'est-à-dire au territoire.

Le transcendantalisme n'est pas, d'un côté, affirmation de la nécessité de créer une littérature et une pensée américaines, et, d'un autre côté, affirmation de l'originalité intrinsèque de tout être humain. II n'est pas, d'un côté, une déclaration d'indépendance philosophique et, de l'autre côté, une pensée de l'infini. Il est à la fois, et d'une manière très particulière, une philosophie de l'indépendance et de l'infini. 
Je crois que, tout compte fait, les transcendantalistes se sont peu interrogés sur ce que l'on pourrait appeler les conditions de possibilité d'une pensée américaine. Ils se sont peu demandé ce que pourrait être cette pensée, s'ils avaient des précurseurs dans les générations précédentes, en quoi une philosophie américaine se distinguerait des philosophies étrangères ou comment elle pourrait s'imposer à côté d'elles. Pourtant, être soi-même est une question qui les a littéralement obsédés. Et c'est ce que j'ai tenté de suggérer ici. À quel point le transcendantalisme a été une réflexion sur le poids des influences, des conventions ou des traditions, sur les entraves qui retiennent l'être, sur le rapport entre le dedans et le dehors. Une réflexion sur la recherche par laquelle l'être cerne sa propre nature, authentique, intérieure; sur ce qui cherche à le limiter, à l'emprisonner, à le définir par des termes autres que les siens; sur le mouvement qui le pousse à s'épancher, à s'envoler vers l'extérieur. La recherche d'un chemin par lequel l'être pourrait accéder au plus individuel et au plus universel, à l'infini du monde et à l'infini qu'il porte en lui-même.

SYLVIE CHAPUT traductrice et écrivaine 